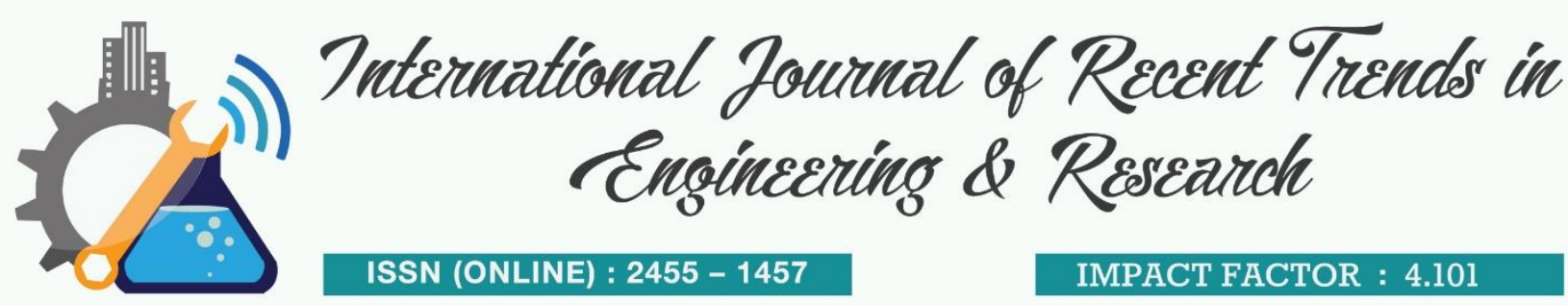

\title{
The Utilization of Cloud Computing as Virtual Machine
}

\author{
Zuhri Ramadhan ${ }^{1}$, Liza Fitriana ${ }^{2}$, Dodi Siregar ${ }^{3}$, Rachmat Aulia $^{4}$, \\ Andysah Putera Utama Siahaan ${ }^{5}$ \\ ${ }^{1,5}$ Faculty of Computer Science, Universitas Pembanguan Panca Budi, Medan, Indonesia \\ ${ }^{2}$ Department of Informatics, AMIK Harapan, Medan, Indonesia \\ ${ }^{3,4}$ Department of Informatics, Sekolah Tinggi Teknik Harapan, Medan, Indonesia \\ ${ }^{5}$ Student of Universiti Malysia Perlis, Kangar, Malaysia
}

\begin{abstract}
Cloud computing has a significant role in the world of information technology, in particular for the retrieval and storage of relevant information. Globally, it creates a more dynamic, fast and precise function. In cloud computing, all information is stored online and does not require hardware like a conventional system. All are available globally on the internet network. It also eliminates the huge cost of buying and managing all existing hardware. Cloud computing has a quick access speed and can be managed in real-time. The data on the server can be easily arranged and distributed to people in need. It will lead the users to use and adapt this technology quickly. This technology generates speed and reliability more than the previous technology.
\end{abstract}

Keywords - Cloud Computing, Information Technology, Virtual Machine

\section{INTRODUCTION}

Cloud computing has become a necessity in the era of technology. The large data users will be increasingly interested in using this technology. The need for extensive data resulting in data storage must be everywhere making it easier for users to access the data [1]. Storage needs are a costly thing to do. To build a data storage server, this requires a huge cost. Online storage and information technology are expected to reduce manufacturing and maintenance costs on server-related devices. As well as with the operating system. The use of virtual machine helps users to lessen the cost of purchasing hardware. This technology has more credibility than conventional technology. So this technology will eventually reach the level of investment in service that is fast and easy. Keep in mind also what exactly is cloud computing and what are the possible risks. In building cloud computing, service providers must understand how to cope with the dangerous possibilities that will occur. Some threats that occur should be anticipated so as not to damage the system located in the virtual machine and data residing on online storage.

\subsection{Cloud Computing}

\section{THEORIES}

Cloud computing is the incorporation of internet technology with online media storage. The real meaning is a use of technology that uses the internet and is controlled from the server. The server can be placed anywhere depending on the service provider. The function of this server is to manage data and applications related to data. Cloud computing helps consumers to use applications in data processing without installing on their devices [2]. They can directly access private files on any computer with internet access. So this technology produces high efficiency by centralizing storage at a place.

Cloud computing is also an abstraction of the hidden complex infrastructure [3]. It is also a computational method in which information technology-related capabilities are presented as a service 
so that users can access it over the internet without knowing what is inside, the expert, or who has control over the technology infrastructure that helps. Cloud computing alters a paradigm in which information is permanently stored on servers on the internet and temporarily stored on a user's computer including desktop, tablet, notebook, personal computer, handheld, sensors, monitors, etc.

There are several characteristics of cloud computing:

1. On-Demand Self-Services. A cloud computing service should be able to be utilized by the user through a self-service mechanism and immediately available when it is necessary. When it needs a service, it can be registered as self-service.

2. Broad Network Access. A cloud computing service should be accessible from anywhere, anytime, by any device. It is connected to the service network. While connected to the Internet, it must be able to access the service through laptops, desktops, internet cafes, mobile phones, tablets, and other devices.

3. Resource Pooling. A cloud computing service should be centrally available and can share resources efficiently. Because various customers share cloud computing, service providers must be able to share loads efficiently, so that the system can be fully utilized.

4. Rapid Elasticity. A cloud computing service should be able to increase or decrease capacity as needed. For example, if employees in the office increased, it should also be able to add users to the application easily. If employees are reduced or placing a news website in a cloud computing network or if there is an increase in traffic because there is important news, the speed and capacity should be upgraded automatically.

5. Measured Service. A cloud computing service should be made available on a scalable basis, as it will be used in the payment process. Keep in mind that cloud computing services are paid for usage, so they must be measurable.

\subsection{Virtual Machine}

A virtual machine is a device that supports the performance of cloud computing. Cloud computing requires good performance and fast. So it takes a backup system that can replace the main system if experiencing constraints at the time of operation. A virtual machine can work just like a conventional operating system. Systems established must be capable of approaching a real system [3]. With this, the device used should be adequate and of good quality. The virtual machine has two terms, guest, and host. The virtual machine does not have a physical part but has a working process like a system that has a physical. The virtual machine is called a guest operating system. This system is simply installed on the computer running it or better known as the host operating system. The basic technique of a virtual machine is to emulate hardware from a single computer consisting of CPU, RAM, HDD to several environments, making it seem like it has the actual device on each of those operating systems [6].

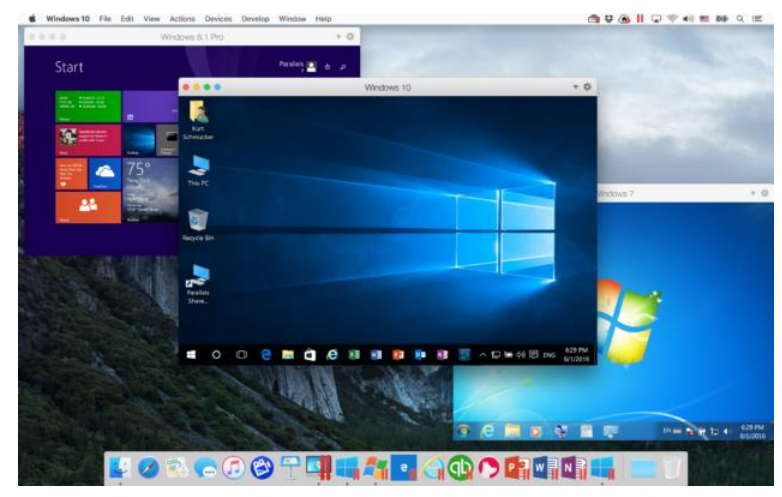

Figure 1. Virtual machine runs three operating systems 
Figure 1 illustrates that the operating system Windows 7, Windows 8 and Windows 10 are running on Mac OSX operating system. The three operating systems act as a guest operating system, while Mac OSX is the host operating system. The four operating systems are running smoothly together. This host operating system performance must be supported by the right hardware as well.

Virtual machine technology has several abilities such as backing up data, run multiple operating systems, system rescue, and running of previous software [4] [5]. One of the most important applications of virtual machine technology is cross platform integration.

Some other important apps performed in the virtual machine platform are:

1. Server Consolidation. If multiple servers run applications that take up only a small amount of resources, the VM can be used to merge them into one server, even if they require different operating systems.

2. Automation and Consolidation. Each virtual machine can act as a different environment; it is usable to the developer, so there is is not necessary to provide the physical environment.

3. Easy System Recovery. Virtualization can perform system recovery that requires portability and flexibility between platforms.

4. Software Demonstration. The virtual machine technology can provide a clean operating system and its configuration.

\section{RESULT AND DISCUSSION}

In the use of cloud computing, users will get from some aspects especially the cost. Usually, some vendors provide virtual operating system rental or data storage online. The amount of capacity obtained depends on the ability of the customer to choose which is more suitable for their activities.

There are several benefits using cloud computing:

1. The start-up cost of this technology is not as expensive as building a physical system.

2. Cloud computing services can be easily upgraded or downgraded as needed.

3. Operational and usage costs will be based on how much capacity and speed desired

4. This service will use multi-tenant method (many customers in 1 platform).

5. The ability to adjust services will be unlimited.

6. Make more flexible, can easily be oriented to profit and rapid development.

7. Make operational and management easier, is possible because the personal system or a company that is connected in a cloud can be easily monitored and arranged

As a service business, cloud computing technology has many risks. Data security is at stake. As long as the data is online, it could lead to data theft by irresponsible people. It does not matter if the data is not so important, but what about if the data is related to the defense of the country, it is more dangerous. If the data is successfully stolen and sold to other countries, this will lead to leakage of state defense and have a significant risk. Before using cloud computing services as a provider of information technology, the risks that may occur should be addressed and avoided.

Some factors to consider before using cloud computing services:

1. Service level. Cloud providers may not be consistent with the performance of apps or transactions. It requires understanding the service level obtained regarding transaction response time, data protection and data recovery speed.

2. Privacy. Because other people/companies also do hosting, the possibility of data can be out or read without the user's knowledge or approve from the user.

3. Compliance. Consider the regulation of the business owned, in this case theoretically Cloud service provider, is expected to equalize the level of conformity for data storage within the 
Cloud, but because this service is still effortless to be expected to be careful regarding data storage.

4. Data ownership. Is the data still user's data once the data is stored in the cloud or the virtual machine? Maybe this question is a little strange, but please note as it happens on Facebook that tries to change terms of use its agreement also question this.

5. Data Mobility. Can a user share data between Cloud services? Moreover, if a user terminates the cloud relationship how to get data back? What format will be used? Alternatively, can a user make sure the copies of the data have been deleted? For a service that is still critical for a company, the best advice is to ask these questions in detail and get all commitments in writing.

After all the factors work well, the next step is to improve the performance of the service. It includes access speed and storage capacity. The most important thing is the speed at which users will use this to download and upload files. The faster the speed offered, the more expensive it will cost to get the service. Data security and convenience are indispensable in this service. This convenience can be obtained from backup data or backup system provided by the virtual machine. So that if one day experienced a constraint, the virtual machine could restore the work of the physical system is stalled.

\section{CONCLUSION}

The use of cloud computing is beneficial to solve the problem of data capacity and convenience. In this usage, the user does not have to worry anymore about the issue of electrical energy problems, speed, and cost of maintenance on the system. Users simply by paying for services within a period, have been getting services at a certain level. This service can also provide the operating system virtually if the user wants to have applications that can run virtually. Virtualization is a method to make an application independent of the system that the user must create.

\section{REFERENCES}

[1] E. Mugisha and G. Zhang, "Reliable Multi-cloud Storage Architecture Based on Erasure Code to Improve Storage Performance and Failure Recovery," International Journal of Advanced Cloud Computing and Applied Research, vol. 3, no. 1, pp. 26-40, 2017.

[2] A. Schwanenge, A. Schönl and C. Linnhoff-Popien, "Location-Based Cloud Resource Allocation Based on Information of the Social Web," International Journal of Advanced Cloud Computing and Applied Research, vol. 1, no. 1, pp. 1-12, 2013.

[3] Hamdani and A. P. U. Siahaan, "Virtualization Approach Theory and Application," IOSR Journal of Electrical and Electronics Engineering, vol. 2, no. 5, pp. 187-191, 2016.

[4] Vijayrajan, "Analysis of Performance in the Virtual Machines Environment," International Journal of Advanced Science and Technology, vol. 32, no. 7, pp. 53-64, 2011.

[5] I. Ali and N. Meghanathan, "Virtual Machines And Networks, Installation, Performance, Study, Advantages and Virtualization Options," International Journal of Network Security \& Its Applications, vol. 3, no. 1, pp. 1-15, 2011.

[6] A. P. U. Siahaan, "Comparison Analysis of CPU Scheduling: FCFS, SJF and Round Robin," International Journal of Engineering Development and Research, vol. 4, no. 3, pp. 124-132, 2016. 